\title{
Catorce Años de Educación Bilingüe en la Amazonía: Análisis y Prospectiva \\ Programa de Educación Bilingüe e Intercultural del Alto Napo
}

\section{Gabriel Ashanga Jota*}

\author{
Rosa E. Vera* \\ Gloria San Román* \\ Lucas Tushupe*
}

This article presents the experiences of the Bilingual and Intercultural Program of the Upper Napo (PEBIAN in Spanish). This program was introduced mainly in the Quichua speaking Napuruna villages and in one Siecoya village of the Napo River, a tributary of the Amazon River in the northern tropical rainforest of Peru.

The program began in 1972 and is based on the cultural traditions of the ethnic Naporuna and with the goal of preserving the identity of these people. This educational system opposes liberation-education to domination-education. One of the principal objetives is that the responsability of the program be turned over to the indigenous bilingual teachers as soon as possible. 
Cet article présente l'expérience du Programme d' Education Bilingue et Interculturelle du Haut-Napo (PEBIAN). Ce pregramme se développe principalement parmi les communautés Napurunas, et aussi dans une communauté Siecoya, des rives du fleuve. Napo, affluent de l' Amazone dans la jungle nord du Pérou.

Le programme fut mis en marche dès 1972, en s'appuyant sur les traditions culturelles de 1 " "ethnie Napuruna, et avec le but de sauvegarder l" identité de ce puple. Le systéme d" enseignement oppose $1^{\prime}$ éducation-libération a $1^{\prime \prime}$ éducation-domination. Un des objectifs principaux vise à ce que le plus tôt posible la responsabilité du programmme se trouve entre les mains de professeurs bilingues indigénes.

\section{La población a la que atiende el programa}

En Programa de Educación Bilingüe e Intercultural del Alto Napo (PEBIAN), se desarrolla en las comunidades Napurunas ubicadas a orillas del río Napo, (afluente del Amazonas en la selva norte del Perú) y en una comunidad Siecoya. Las lenguas maternas de los educandos son el Kichwa y Siecoya.

\section{Pueblo Napuruna.}

El pueblo Napuruna o Sunu, ordinariamente es llamado Kichwa del Napo por el idioma que le unifica. Este idioma es el Kechwa en su variedad Napeño Inga.

Los napurunas del Perú forman 35 comunidades con una población total de 12,000 personas. (Los caseríos están muy distantes unos de otros y los asentamientos de cada familia dentro de una misma comunidad están en una forma dispersa). 
Fl pueblo Napuruna ha sufrido la esclavitud. Los encomenderos primero y más tarde los patrones y regatones, los manipularon dismuyendo su capacidad de organización y liderazgo.

Loa patrones del Alto Napo con astucia observaron la vida y creencias de los napurunas, calcularon su punto vulnerable y por él les acometieront: El compadrazgo. El compadrazgo adquirido por apadrinamiento en matrimonio o bautismo, crea entre el los un lazo familiar al que se debe permanecer fiel. De esta fidelidad usó y abusó el patrón; con e! parentesco tenía un pretexto para exigir fidelidad y les podía dominar.

El patrón proporcionó trabajo de explotación de recursos naturales por un salario ínfimo o bien habilitaba a los runas, adelantándoles plata que nunca terminaban de pagar. La presencia del patrón en una comunidad napuruna ha sido una amenaza permanente. El patrón se establecía en la comunidad como un quiste que corroe la pacífica convivencia.

El regatón es el comerciante de los ríos. Ofrece productos a precios elevados y con.sus actitudes es otro elemento distorsionador del pueblo.

Frente a esta situación, en el año 1972 han comenzado a organizarse.

En la actualidad, de acuerdo con la Ley de Comunidades Nativas, cada comunidad tiene su autoridad propia: Apu (Jefe), promotor de salud, profesores bilingües y otros encargados de distintos servicios.

Los napurunas crean la Federación Kichwaruna Wangurina (ORKIWAN) en 1978 con el fin de defenderse como personas y como pueblo. Hoy en 1989 podemos decir que se han liberado totalmente de los patrones y que desde ORKIWAN, de una manera todavia incipiente, etán resurgiendo como pueblo con identidad propia, en diálogo con los otros pueblos que conforman el Perú.

Como ya hemos dicho, el PEBIAN también desarrolla su trabajo en una Comunidad Siecoya: Resto del gran Pueblo de Encabellados, masacrados por los patrones, encomenderos y caucheros; son hombres orgullosos de su cultura e idioma, sin complejo de inferioridad y amantes de la libertad.

La comunidad Siecoya del Napo era más numerosa; pero hace unos diez años han pasado muchos al Ecuador. En la actual idad está formada por 7 familias. Desde 1982 tienen escuela bilingüe atendida por el PEBIAN.

\section{Antecedentes del programa}

La educación regular en el Alto Napo comienza en 1964 con la creación de escuelas con profesores mestizos (venidos de fuera), en algunas comunidades. Hay buena intención en 
hacer llegar maestros a lugares tan apartados. Hay también una gran equivocación, la de ignorar que el pueblo Napuruna tiene una cultura, un idioma y una manera propia de educar a sus hijos.

Con esta actitud base se inicia un proceso educativo que distorsionó los ejes básicos del desarrollo del niño napuruna. Si antes era educado por sus padres dentro de los hechos de la vida diaria, a partir de este momento se le impuso una educación que pretendía enseñarle creando situaciones artificiales e irreales para él. El niño napuruna que había aprendido haciendo, tendrá que aprender memorizando palabras que no entiende.

Pronto los profesores llegados de fuera se colocaron del lado de los patrones que estaban explotando al pueblo. El idioma Kichwa según ellos debería ser erradícado. Así, día tras día, se sembró en los niños el desprecio a lo suyo, que debían olvidar para aprender sólo lo que se les traía de fuera. Pronto se vio el fruto de esta educación al margen de las coordenadas lingüístico culturales y sicológicas del pueblo: serios problemas de aprendizaje, falta de promoción, escasa presencia del alumnado femenino, analfabetismo e infravaloración de la propia cultura.

Simultáneamente en la zona desde 1971, el P. Juan Marcos Mercier y otros venían trabajando en el análisis de la realidad, la investigación lingüística, la fijación por escrito del idioma y la recopilación de mitos e historia del pueblo napuruna. Tudos estos datos fueron decisivos a la hora de enfrentar una alternativa a la problemática en el campo de la educación.

A partir de 1973 se comenzaron a producir materiales de lectura y escritura en Kich wa y a entrenar para su aplicación a maestros mestizos de servicio en la zona. Después de dos años de trabajo en este sentido se decidió, a petición de las mismas comunidades, preparar a jóvenes nativos napurunas que habian terminado su primaria, para ser maestros de su pueblo. Es así como en 1975 se inicia el Programa de Educación Bilingủe e Intercultural del Alto Napo en dependencia directa del Ministerio de Educación, a través de la Sexta Región de Educación de Loreto y con el apoyo inicial del Vicariato "San José del Amazonas".

\section{Creación del programa}

Los abusos de los profesores foráneos y el deseo de afirmarse como personas con características propias, es lo que movió al pueblo napuruna a pedir una educación diferente y acordecon lo suyo.

El conocer la cultura y la situación histórica a la que habían sido llevados por las acciones negativas referidas, ofrecía una certeza a la hora de iniciar el trabajo: La educación habria de surgir desde dentro; desde la cultura, el momento Histórico y la vida de aquellos a los que se dirigía. 
Se conocía ya la manera de ser de este grupo. Habían rasgos bien definidos en su cultura e historia que marcaban el camino:

- Los runas tienen conciencia de Pueblo y como tal había que alcanzarles los medios para su autodeterminación.

- La lealtad hacia su etnia, injustamente debilitada por factores externos, sería reforzada y potenciada.

- Si los mayores han transmitido siempre el saber, los mayores, los primeros que fueron a la escuela, deberían enseñar a los otros. Desde el inicio se vio claro que los profesores bilingües deberian ser nativos.

- Los napurunas tienen una manera de enseñar a sus hijos desde la vida y para la vida desde su propio medio.

- El compartir es la raíz profunda de sus valores familiares y grupales, en comunidad y para la comunidad habia que educarles.

La política educativa del momento era propicia. Las declaraciones de la UNESCO habían provocado una toma de conciencia hacia la educación de los diferentes grupos étnicos. La Ley General promulgada en 1972 dedicaba varios de sus artículos a establecer las bases para una educación adecuada a las necesidades y culturas de las distintas etnias:

Art. 12 "La educación se adecuará a los requerimientos locales, zonales, regionales y nacionales y evitará toda forma de imposición cultural..."

Art. $12^{2}$ "La educación considerará en todas sus acciones, la existencia en el país de diversas lenguas que son medios de comunicación y expresión de cultura y velará por su preservación y desarrollo..."

La Ley esta vez respondia al anhelodel Pueblo. Apoyados en ella el P. Juan Marcos Mercier y la Pedagoga y Sicóloga María del Carmen Fernández, presentaron a las autoridades educativas competentes, para su aprobación, el Proyecto de Educación Bilingüe e Intercultural del Alto Napo. Una vez logrado, era este un paso decisivo para la revalorización de un idioma y cultura tanto tiempo marginados.

\section{Fundamentación antropológica del PEBLAN}

Nosotros consideramos la realidad plurilingüe y pluricultural del Perú como una riqueza a la vez que un reto. Desde esta concepción pretendemos reafirmar la identidad del pueblo Napuruna y del pueblo Siecoya sin aislarnos del contexto nacional. 
Entendemos que, potenciando lo genuino de cada cultura y abriendo posibilidades al enriquecimiento con los valores de otras culturas, estamos contribuyendo a formar la auténtica identidad peruana.

Para nosotros, la Educación Bilingüe Intercultural supone una educación basada en los valores, filosofía y educación indígenas; que puede incorporar críticamente elementos válídos de otras culturas.

Sin embargo, el PEBIAN no se limita a revalorizar el aspecto lingüístico cultural, sino que también pretende ayudar al cambio estructural necesario que termine con la marginación secular del indígena. Nos proponemos que la Educación cum pla con la tarea de facilitar la información, análisis, expresión y toma de decisiones de los pueblos Napuruna y Siecoya. De ahí nuestra insistencia en una ed ucación crítica y liberadora, que incentive en el profesor y en el educando el compromiso con el destino de su pueblo.

El equipo coordinador y los docentes del PEBIAN, trabajamos convencidos de estar desarrollando una estrategia que el país necesita, por ser la que más contribuye a consolidar un Perú unido, no erróneamente unificado.

\section{¿Qué es y cómo desarrolla su trabajo el PEBIAN?}

\section{Finalidad del programa} 1975:

EI PEBIAN a través de su trabajo educativo tiene como finalidad desde su creación en

1. "Formar hombres críticos y libres que valorando su propia cultura sean capaces de comunicarse y dialogar con otras culturas sin perder su propia identidad". (Doc. de función del PEBIAN. Iquitos, 1975).

2. Ayudar al cambio estructural necesario para terminar con la marginación del indígena. (Art. $6^{\circ}$ R.I. PEBIAN). 


\section{Objetivos del programa}

Son objetivos del PEBIAN:

1. Crear una educación abierta a la comunidad con participación e integración constante de la misma.

2. Elaborar un modelo educativo en continuidad con la educación tradicional indígena con el fin de recuperar el dinamismo y creatividad de la propia cultura.

3. Crear las estrategias educativas que permitan al indígena dialogar con otras culturas, sin perder su propia identidad.

4. Capacitar a profesores indígenas bilingües para el servicio de sus comunidades, identificados con la mística y finalidad del PEBIAN.

5. Elaborar y publicar libros de texto y folletos específicamente diseñados para el PEBIAN, en un esfuerzo constante de adaptar los contenidos curriculares.

6. Ofrecer al país una alternativa de autofinanciación a través de:

- La utilización responsable de los recursos económicos que la comunidad local, regional y nacional destinan a la educación.

- Aporte voluntario de todos los miembros del PEBIAN como signo de solidaridad con la situación del país y para asegurar la realización y continuidad del Programa. (Art. $7^{9}$ R.I. PEBIAN).

\section{Contenidos y metodología}

En nuestro sistema de enseñanza, a la educación-dominación se opone la educaciónliberación. Desde la conciencia domesticada pretendemos llegar a la conciencia liberada.

Buscamos una educación bilingüe e intercultural que, utilizando su idioma y contenidos culturales ayude al pueblo a organizarse y fortalecer su identidad étnica. Al mismo tiempo que los contenidos del currículo oficial, seleccionamos aquellos que decididamente ayudan a nuestros alumnos a sentirse más seguros y establecer un diálogo crítico con el entorno en todos sus ámbitos.

Entendemos que una escuela al servicio de un grupo étnico, hoy en América Latina, debe desarrollar un currículo diferenciado que, impartido con una metodología y pedagogía indígenas, contribuya a eliminar la marginación de siglos.

Al respecto nos dicen hoy los profesores bilingües del PEBIAN: 
"Someternos acríticamente al currículo oficial, aunque fuera impartido en nuestro idioma; sería caer en la trampa de someternos una vez más, y ahora voluntariamente a la dominación y explotación de los que nos siguen gobernando dentro y fuera del país". (Ponencia elaborada por los profesores bilingües del PEBIAN para el "I Encuentro de Profesores Indígenas del Amazonas en Roraima del Brasil", octubre, 1988).

\section{Formación de profesores}

Como respuesta a la petición de los padres de familia de que se preparen a los jóvenes de la propia etnia para llegar a ser profesores, y por la convicción de que el indígena bien preparado es el mejor maestro de su pueblo, el PEBIAN ha centrado gran parte de su esfuerzo en la formación de los profesores indigenas.

Esta capacitación permanente se realiza a través de:

- Curso de capacitación anual (de 30 a 40 días en el verano).

- Concentración durante las vacaciones de mitad de ar̃o (de 5 a 10 días).

- Círculos de estudio en fines de semana para la evaluación y programación del trabajo, el asesoramiento a los estudios personales e impulsar la vida del Programa (cada mes).

- Asesoramiento personalizado a cada docente en la sede del PEBIAN o mediante las visitas que los especialistas, con una frecuencia de 3 a 5 veces al año, realizan a cada centro educativo.

En el proceso de capacitación y seguimiento se atiende los aspectos de:

- Seria implementación técnico-pedagógica.

- Asesoramiento en los estudios personales.

- Creación de una mística de grupo. De grupo que valora y potencia lo propio, que no busca ganar prestigio ni medrar. De grupo que quiere servir y potenciar a su pueblo para que él mismo sea el gestor de su propio desarrullo.

EI PEBIAN trabaja con la perspectiva de que, a mediano plazo, toda la responsabilidad del Programa pueda ser llevada por los profesores bilingües. Esto obliga a una búsqueda constante de estrategias que incentiven su preparación profesional y su participación al máximo, tanto en la elaboración de materiales didácticos como en todo lo relacionado con el desarrollo del Programa. 
Además de estas acciones que se realizan en el medio, durante el verano, gran parte de los docentes del PEBIAN acuden a la ciudad de Iquitos para seguir los cursos de profesionalización en el Instituto Superior Pedagógico Loreto o en la Universidad Nacional de la Amazonía Peruana.

\section{Producción de materiales educativos y didácticos}

La investigación de la educación tradicional, la cosmovisión, la historia, la tradición oral, la lengua y los factores sociológicos condicionantes, sirven de base para la elaboración de materiales educativos especificos para las escuelas del PEBIAN.

Hasta el momento se han elaborado:

- Cuaderno de aprestamiento "Hawina".

- Libro de lecto escritura inicial en Kichwa "Ayllu Kanchi" (2 ediciones)

- Segundo libro de lectura, con iniciación al castellano "Ñukapa allpa" con ilustraciones hechas por niños Napurunas.

- Libro de lectura bilingüe para los últimos grados "Napuruna rimay". Recoge en Kichwa y en castellano la cosmovisión y la historia del Pueblo Napuruna.

- Folleto para las organizaciones comunales "Imapa wankurinchi".

- Guía del profesor bilingüe "Yachachi rikuchina".

- "Diwswa runawa". Libro que contiene la espiritualidad del Pueblo Napuruna.

- Lecturas "Sumakta yuyarisa", recoge creaciones de profesores y alumnos.

- Orientaciones para la enseñanza de la religión.

- Guía metodológica para la enseñanza de la matemática inicial.

- Guía para la enserianza del Castellano como segunda lengua, con desarrollo de las unidades.

- Guía metodológica de lecto escritura.

- "Sacha hanpikuna". Folleto de cantos con tonos típicos napurunas y adaptación de tonos universales.

- Libro de lectura inicial en Kichwa "Kushi Kanchi". 
Con la participación de profesores y alumnos se elaboran variedad de materiales didácticos a partir de los recursos del medio: materiales de aprestamiento, maquetas, mapas, etc.

\title{
¿Qué entendemos hoy por educación bilingüe e intercultural?
}

\author{
Creemos que una de las características del PEBIAN es su actitud de contrastey reajuste \\ permanente.
}

En este proceso fue significativa la auto-evaluación que hicimos a raíz del "Seminario sobre políticas y estrategias educativo-culturales con poblaciones indígenas" (UNESCO, Lima, 1985) precisamente cuando se cumplian los diez aflos de vida del PEBIAN.

En esa oportunidad, en diálogo y contraste con otros especialistas de América Latina, encontramos como positivo que el grupo Napuruna hubiese recobrado la conciencia de Pueblo; se había liberado de los patrones, se había unido y organizado. (La vergüenza de manifestar sus expresiones culturales ante los de fuera iba desapareciendo lentamente. Valoraban más su idioma, habían recuperado palabras perdidas e incluso habían creado otras, desde su forma de pensamiento). En relación a la Escuela, los problemas que se daban con el profesor mestizo habían disminuido considerablemente.

Sin embargo en esa evaluación encontramos dos hechos que nos preocúpaban:

- El desarrollo cognoscitivo del niño había mejorado, pero no tanto como esperábamos.

- El grado de bilingüismo de los alumnos al terminar la primaria, no nos parecía del todo satisfactorio.

El análisis de estos hechos nos llevo a planificar algunas investigaciones, ya en curso, sobre: "Estilos cognoscitivos, diferencias culturales y educación en el Pueblo Napuruna de la Amazonía Peruana" y, sobre "La formación del concepto en el Napuruna".

Por otra parte vimos llegado el momento de cuestionar nuestro concepto de Educación Bilingüe e Intercultural planteando una nueva alternativa.

"Transmitir durante toda la educación primaria todos los contenidos de las diferentes áreas en el idioma materno y con sus formas de pensamiento propias.

Paralelo a esto, impartir enseñanza del castellano como segunda lengua, dedicándole el tiempo necesario para que el alumno termine la primaria con un bilingüismo satisfactorio".

Después de haber experimentado durante tres años este nuevo enfoque de Educación Bilingüe, podemos decir que los resultados son bastantes satisfactorios en relación a los 
hechos que nos preocupaban. Esto nos está suponiendo un gran esfuerzo de elaboración de nuevos materiales y de creación de términos en lengua vernácula.

\section{Andilisis y reflexión sobre la experiencla}

\section{Logros más significativos del programa}

Logros en relación a la participación de las comunidades en el desarrollo del PEBIAN:

- Los padres de familia se encargan de la construcción del centro educativo, del mobiliario y de la casa del profesot.

- Los padres de familia y alumnos a lo largo del afto confeccionan artesanias que se venden en la ciudad de lquitos y con el importe, se compran útiles escolares. Esto suponeautonomia económica del centro educativo y revitalización de la expresión artística del Pueblo.

- EI PEBIAN es asumido por la Organtzación Kichwaruna Wangurina "ORKIWAN" como el servicio educativo más adecuado a las comunidades napurunas. Dicha organización participa de modo definitivo en las decisiones fundamentales del PEBIAN.

Logros en relación a la cappacitación y compromiso de los docentes indigenas

- Después de catorce afios de trabajo vemos como un acierto que el PEBIAN ha logrado mantener en los profesores nativos la motivacion para superarse en los estudios personales, en la preparación técnico-pedagógica y en la actitud de servicio a su pueblo. El PEBIAN evalúa el trabajo del profesor dentro y fuera del aula.

- Consideramos muy positivo que el Programa, desde su inicio, haya tenido la autofinanciación económica a través del aporte voluntario de un porcentaje de los haberes (actualmente es el $20 \%$ ) de todos los docentes que tenemos nombramiento oficial y de la utilización responsable de los recursos económicos que la comunidad local, regional y nacional destinan a la educación. Esta formula ha garantizado en todo momento la continuidad del Programa.

Cada afio la Asamblea anual del PEBIAN elige democráticamente al equipo de profesores bilinglies responsables últimos de la economía del Programa.

\section{DIGITALIZADO EN EL CENDOC - CAAAP}


- Es idea original del PEBIAN lo que llamamos "Profesor Auxiliar". Durante un periodo de dos a tres affos el joven que aspira a ser profesor bilingüe tiene la oportunidad de trabajar, junto a un profesor ya nombrado, en una escuela del Programa. De este modo el PEBIAN puede evaluar sus capacidades y actitudes para la tarea docente, al mismo tiempo que le prepara antes de proponer su nombramiento oficial.

El profesor auxilar desde su ingreso en el Programa recibe un sueldo procedente del aporte económico de los nombrados.

- Las personas no indígenas que aún estamos en el Programa vivimos en una de las comunidades nativas de la zona, compartiendo nuestra vida y sueldo. Desde la sede del PEBIAN en Angoteros, el acompañamiento a los profesores es cercano y continuo. Como ya hemos dicho trabajamos con la perspectiva de que a mediano plazo todo el Programa sea asumido por los profesores indigenas. En la actualidad dos profesores nativos forman parte ya del equipo coordinador con nombramiento oficial.

- Es preocupación constante del PEBIAN el que los profesores nos mantengamos en una actitud de solidaridad con el país y abiertos al díalogo crítico con otras culturas. En este sentido es significativo que:

- En los cursos de capacitción invitemos a especialistas competentes para que nos ayuden a reflexionar y a tomar postura frente a la problemática nacional.

- Cada profesor esté suscrito a KANATARl, periódico semanal en línea de concientización y denuncia.

- En 1986, con nuestros ahorros, 23 profesores del Programa viajamos a la sierra del Perú para hacer una experiencia de intercambio cultural con los indígenas de la provincia de Anta del departamento de Cusco.

- A pesar de las dificultades geográficas y económicas, el PEBIAN procura estar presente en eventos regionales, nacionales e internacionales referentes a educación bilingüe y a la defensa de la causa indígena. En el mes de octubre del año en curso, un profesor nativo del PEBIAN a solicitud del CIMI, ha presentado la experienciadel PEBIAN en un encuentro de profesores bilingües en Manaus (Brasil).

\section{Dificultades que repercuten en el trabajo del Programa}

La problemática que hemos tenido que afrontar, se ha ido formulando con mayor nitidez a lo largo de los catorce años de experiencia. 
Creemos que el principal eje de esta problemática gira en torno a la identidad. Los pueblos napurunas y siecoya poseen una fuerte cohesión social y una clara identidad; son pueblos que se saben distintos. En efecto, la historia de opresión y el rechazo y marginación que vienen soportando no les ha impedido transmitir su cultura de generación en generación mediante su idioma y costumbres.

El problema no está en la falta de identidad sino en la negación, en el no reconocimiento de su identidad de parte de la sociedad envolvente. Sociedad que arrastra desde tiempo atrás desconocimiento y prejuicios acerca de los pueblos nativos de tal manera que no les permite un espacio en el ámbito global, ni intuye lo que desde su modo de ser pueden aportarle.

Esta realidad nacional crea en los padres de familia, e incluso en algunos profesores, sentimientos de inseguridad y desconfianza respecto al Programa. Se ha dado el caso de que alguna comunidad después de haber solicitado educación bilingủe la ha rechazado aun antes de haberse iniciado el trabajo por la influencia de ciertos comerciantes y funcionarios.

Otro de los ejes de la problemática está relacionado con la carencia de un marco legal definido de la Educación Bilingüe en el Perú. La reciente Dirección General de Educación Bilingüe, recién ha definido oficialmente su política.

Por esta realidad, a pesar de tener el reconocimiento legal desde 1975, el PEBIAN sigue soportando la falta de comprensión y continuas trabas por parte de algunas autoridades educativas inmediatas, que se mueven con los esquemas de la educación nacional indiferenciada y por tanto identifican currículo alternativo con empobrecimiento.

En el verano, bastantes profesores del PEBIAN siguen cursos de profesionalización en Iquitos. Sin embargo la falta de una profesionalización específica bilingüe en el departamento es una dificultad: No ayuda a fortalecer la identidad indígena de los docentes nativos, y más bien contribuye a reforzar el Sistema al no ofrecer un modelo de escuela diferenciado que sirva a la liberación de los pueblos indígenas.

\section{Prospectiva}

Frente a la complejidad e indiscutible interés de esta problemática a la hora de plantearnos la prospectiva lo hacemos en una doble dimensión:

- Desde el trabajo del PEBIAN vemos la urgencia de reforzar la seguridad de las comunidades y de los profesores, para que sean más conscientes de las trampas que la sociedad envolvente les pone, haciéndoles creer que siendo como ellos van a progresar, cuando en realidad lo que buscan es que pierdan la identidad para seguir sometiéndolos a sus intereses. 
- Hacia fuera reivindicamos el derecho de los pueblos indígenas a ser reconocidos y. respetados como ciudadanos íntegros en la teoría y en la práctica, sin que para ello se les obligue a renunciar a su propia identidad indígena.

Desde aquí hacemos un llamado a políticos, investigadores y educadores para una reflexión seria sobre la realidad multilingüe y pluricultural del país, y sobre la necesidad de elaborar un modelo educativo que conduzca a una identidad nacional en la que tengan cabida igualitaria todas las culturas del Perú.

En este modelo educativo consideramos urgente propiciar los curriculos diferenciad os para la variedad de culturas vernáculas. Al mismo tiempo entendemos que el currículo actual para la sociedad envolvente debe ser rectificado en el sentido de corregir su racismo y enfoque parcial respecto a la realidad plural del país.

Consideramos también que los medios de comunicación social tienen gran responsabilidad en la tarea de erradicar el racismo en el Perú, ya que ellos influyen poderosamente sobre el pueblo.

Nos parece llegado el momento de que el encuentro de culturas no sea resuelto, como tantas veces lo ha sido en la historia, mediante una cómoda y aplastante absorción.

El aportar por la injusta uniformidad o el difícil pero enriquecedor diälogo en la pluralidad, es un reto que se nos plantea hoy en el Perú y en los otros paises de América Latina.

Nuestro modelo de educación bilingüe e intercultural se propone contribuir y' acompaftar al proceso de mantenimiento, desarrollo y diálogo de todas las culturas. La tarea no es fácil, la educación bilingüe e interculturai no puede afrontar el reto aisladamente, es obra de todos los sectores públicos y privados; es obra de todos y cáda uno de los que optamos por un Perú unido pero no uniformado. 